\title{
SLOWLY-MIGRATING TRANSITION LAYERS FOR THE DISCRETE ALLEN-CAHN AND CAHN-HILLIARD EQUATIONS
}

\author{
CHRISTOPHER P. GRANT* AND ERIK S. VAN VLECK ${ }^{\dagger}$
}

\begin{abstract}
It has recently been proposed that spatially discretized versions of the Allen-Cahn and Cahn-Hilliard equations for modeling phase transitions have certain theoretical and phenomenological advantages over their continuous counterparts. This paper deals with one-dimensional discretizations and examines the extent to which dynamical metastability, which manifests itself in the original partial differential equations in the form of solutions with slowly-moving transition layers, is also present for the discrete equations. It is shown that, in fact, there are transition-layer solutions that evolve at a speed bounded by $C_{1} \varepsilon\left(1+C_{2} /(n \varepsilon)\right)^{-C_{3} n+C_{4}}$ for all $n \geq n_{0}$ and $\varepsilon \leq \varepsilon_{0}$, where $1 / n$ is the spatial mesh size, $\varepsilon$ is the interaction length, and $n_{0}$ and $\varepsilon_{0}$ are constants.
\end{abstract}

Key words. Cahn-Hilliard equation, Allen-Cahn equation, lattice differential equations, metastability

AMS subject classifications. 35B30, 34K25

Abbreviated title. Slowly-Migrating Transition Layers

1. Introduction. Two important partial differential equations for modeling the formation and motion of phase boundaries are the Allen-Cahn equation [4]

$$
\begin{gathered}
u_{t}=\varepsilon^{2} \Delta u-f(u), \quad x \in \Omega \\
\partial_{\nu} u=0, \quad x \in \partial \Omega,
\end{gathered}
$$

and the Cahn-Hilliard equation [11], [13]

$$
\begin{gathered}
u_{t}=-\Delta\left(\varepsilon^{2} \Delta u-f(u)\right), \quad x \in \Omega \\
\partial_{\nu} u=\partial_{\nu} \Delta u=0, \quad x \in \partial \Omega .
\end{gathered}
$$

In both equations, $\Omega$ is a bounded domain, $\varepsilon$ is a small positive constant called the interaction length, and $f(u)$ is the derivative of a potential function $W(u)$ that has two wells of equal depth. For concreteness and simplicity, we will assume that $W(u)=\left(u^{2}-1\right)^{2} / 4$, but results similar to those obtained in this paper may be obtained for qualitatively similar $W$.

In this paper, we will study the following systems of ordinary differential equations that are obtained by spatially discretizing (1.1) and (1.2) when $\Omega=(0,1)$ :

$$
\begin{gathered}
\dot{u}_{i}=\nu^{2}\left(u_{i-1}-2 u_{i}+u_{i+1}\right)-f\left(u_{i}\right), \quad i=0, \ldots, n \\
u_{-1}=u_{0}, u_{n}=u_{n+1}
\end{gathered}
$$

and

$$
\begin{array}{cc}
\dot{u}_{i}=-n^{2}\left(\mu_{i-1}-2 \mu_{i}+\mu_{i+1}\right), & i=0, \ldots, n \\
u_{-1}=u_{0}, u_{n}=u_{n+1} & \\
\mu_{i}=\nu^{2}\left(u_{i-1}-2 u_{i}+u_{i+1}\right)-f\left(u_{i}\right), & i=0 \ldots n \\
\mu_{-1}=\mu_{0}, \mu_{n}=\mu_{n+1} . &
\end{array}
$$

\footnotetext{
* Department of Mathematics, Brigham Young University, Provo, Utah 84602 USA.

$\dagger$ Department of Mathematical and Computer Sciences, Colorado School of Mines, Golden, Colorado 80401 USA. The work of this author was supported in part by NIST grant \# 60NANB4D1698.
} 
Here, each $u_{i}$ is a function of the time $t$, and " " represents differentiation with respect to $t$. Also, $1 / n$ is the spacing between meshpoints, and $\nu=n \varepsilon$. Although we consider the discrete analog of Neumann boundary conditions in (1.3) and (1.4), similar results may be obtained for other boundary conditions.

The particular phenomenon we wish to address here is the slow migration of phase boundaries. For both (1.1) and (1.2), solutions corresponding to small inhomogeneous perturbations from $u=0$ will typically develop sharp transition layers separating regions where $u$ is close to -1 from regions where $u$ is close to +1 (see, e.g., [15], [19], [27]). Subsequently, these layers slowly migrate. When $\Omega$ is one-dimensional the layers move at a speed that is $O\left(e^{-C / \varepsilon}\right)$ as $\varepsilon \rightarrow 0$, where $C$ depends only on the distance between layers [1], [5], [14], [25], [26], [28]. For higher-dimensional $\Omega$, the speed of migration remains exponentially small for (1.2) if the interfaces are nearly circular [2], [3], but is only $O\left(\varepsilon^{2}\right)$ for (1.1) because motion is then driven by curvature [9], [15], [18], [24], [31]. These slowlymoving solutions are interesting because, although far from any true equilibrium state, they may easily be mistaken for such. This behavior is called dynamical metastability by Bronsard and Kohn [8] and dormant instability by Fusco and Hale [26].

It is natural to ask to what extent slow motion is preserved when the space variable is discretized. The following results are known:

1. For $\varepsilon$ fixed, as $n \rightarrow \infty$, the speed of transition layers (on finite time intervals) becomes asymptotically of order $e^{-C / \varepsilon}$ (see [23]).

2. For $n$ fixed, if $\varepsilon$ is sufficiently small then any transition-layer state is close to a (true) stable equilibrium, so no significant motion occurs (on any time scale) (see [22], [29]).

Our contribution is to show that the slowness of the motion in equations (1.3) and (1.4) can be estimated in terms of $\varepsilon$ and $n$, within certain (independent) bounds on those parameters. Our results do not depend on the available results for the corresponding PDEs, but we have made use of techniques which were originally invented to handle PDEs but which we have been able to interpret abstractly in a way that makes them applicable to the discrete equations directly.

We have chosen to study these discrete equations for several reasons. The first reason is that the equations represent a simple scheme that might be used to simulate (1.1) and (1.2) numerically. (However, we make no attempt to match the slowness of the motion for (1.3) and (1.4) to that for (1.1) and (1.2).) The second reason is that these spatially discrete equations often exhibit extremely robust phenomena that are not present in the corresponding PDEs (see for example [12]). Third, they are of theoretical interest in their own right because the physical phenomena being studied are actually particulate in nature; indeed, the partial differential equations are often derived as continuum approximations of discrete systems (see [10], [17] and [30] for examples in the material sciences).

In the biological sciences, equations of the form (1.3) on an infinite lattice have been proposed as a model for propagation of nerve pulses in myelinated axons and have been addressed by several authors [6], [7], [16], [32], [35], [36], and [37]. In particular, the existence and stability of traveling wavefronts have been studied for a nonsymmetric double well potential $W$. Although the mechanisms driving wavefronts on finite and infinite lattices seem fundamentally different, there is the following connection between the two cases: Using summation by parts, it can be shown that when $W$ satisfies the equal-well condition we required, any wavefront is, in fact, stationary, and a truncation of such a stationary wave is utilized in Section 4 of the present paper.

In order that our results may be stated precisely, we introduce some notation and 
terminology. Given a continuous function $u:[0,1] \rightarrow \mathbf{R}$, let $Z(u)$ be the set of zeros of $u$; if $u$ is an element of the $(n+1)$-dimensional vector space of real column vectors

$$
U_{n}=\left\{\left(u_{0}, u_{1}, \ldots, u_{n-1}, u_{n}\right)^{T}: u_{i} \in \mathbf{R}\right\},
$$

let $Z(u)$ be the set of zeros of the piecewise linear interpolation of the data $\left\{\left(i / n, u_{i}\right): i=\right.$ $0, \ldots, n\}$. Let $d(\cdot, \cdot)$ be the Hausdorff distance between sets; i.e.,

$$
d(A, B)=\max \left\{\sup _{a \in A} d(a, B), \sup _{b \in B} d(b, A)\right\} .
$$

Let $\tilde{v}:[0,1] \rightarrow\{-1,+1\}$ be piecewise constant with precisely $k$ discontinuities $\left\{x_{1}, x_{2}, \ldots, x_{k}\right\} \subset(0,1)$, and let $v \in U_{n}$ be its spatial discretization, i.e., $v_{i}=\tilde{v}(i / n)$. Let $r$ be the largest number such that $\left(x_{j}-r, x_{j}+r\right) \subset(0,1)$ for $1 \leq j \leq k$ and $\left(x_{j}-r, x_{j}+r\right) \cap\left(x_{\ell}-r, x_{\ell}+r\right)=\emptyset$ for $1 \leq j<\ell \leq k$.

(Throughout this paper $v, \tilde{v}, r$, and $k$ will have the meanings presented here.)

Then our main result is the following:

THEOREM 1.1. There exists $n_{0}=n_{0}(\rho)$ and $\varepsilon_{0}=\varepsilon_{0}(\rho, k)$, and discretized initial data $u(0)$ within $O(\sqrt{\varepsilon})$ of $v$ that generate solutions $u(t)$ to (1.3) and (1.4) with transition layers moving so slowly that the time necessary for $d(Z(v), Z(u(t)))$ to exceed a fixed value $\rho \leq r$ is greater than

$$
\frac{1}{C_{1} \varepsilon}\left(1+\frac{C_{2}}{n \varepsilon}\right)^{C_{3} n-C_{4}}
$$

for $n \geq n_{0}$ and $\varepsilon \leq \varepsilon_{0}$. Here, $C_{1}, C_{2}, C_{3}$, and $C_{4}$ depend on $r, k$, and $\rho$, but not on $n$ or $\varepsilon$.

The basic approach that is employed to prove this theorem is based on the variational method developed by Bronsard and Kohn [8] for studying (1.1) and incorporates ideas introduced in [28] that, for the continuous equations, yield exponential, rather than superpolynomial, estimates. (For the discrete equations, these exponential estimates are recovered in the limit as $n \rightarrow \infty$.) We note that the results for the continuous case have been of no direct use to us in the discrete case, since the metastability estimates are subtle enough that they can easily be overwhelmed by discretization error. The relevant estimates seem more difficult to obtain for the lattice equations than for the PDEs because, among other reasons, two parameters must be accounted for rather than just one, and a convenient asymptotic "energy" formula (see, e.g., [34]) is unavailable.

Slow motion has been observed numerically for the Allen-Cahn and Cahn-Hilliard equations in [14], [21], [22] and [33]. Using the Lyapunov function presented in Du and Nicolaides [20] for a full discretization (spatial and temporal), Estep [23] has shown that a temporal discretization of (1.3) inherits the slow motion property for sufficiently fine discretizations. Our results show that transition-layer motion must, in some sense, be slow for (1.3) and (1.4) without requiring a discretization whose fineness depends on $\varepsilon$. In other words, there is a neighborhood of the origin in the first quadrant of the $(\varepsilon, 1 / n)$-plane throughout which the speed of interfaces can be estimated.

It is possible for a time varying solution of (1.1) or (1.2) to become an equilibrium solution of (1.3) or (1.4). This phenomenon is sometimes called pinning in the materials science literature, and has been studied for these types of equations by Cahn [10] and others. It is desirable to complement our main result by one which describes what must 
happen before interfaces can become pinned. While not, by any means, giving a complete resolution to this problem, the following result yields some information about the spacing of interfaces in a pinned solution to the discrete equations. In the presentation of this result, we will say $u_{i}$ is a local maximum (minimum) if $u_{i} \geq(\leq) u_{j}$ for all lattice sites $j$ adjacent to $i$.

THEOREm 1.2. Let $u \in U_{n}$ be a nonconstant equilibrium of (1.3) or of (1.4), and let $u_{i}$ and $u_{j}$ be a local minimum and a local maximum, respectively. If $\nu \geq 1 / \sqrt{2}$ then

$$
|i-j| \geq\left\lfloor\frac{\pi}{\cos ^{-1}\left(1-\frac{1}{2 \nu^{2}}\right)}\right\rfloor-1 .
$$

Consequently, the transition layers of a solution u to (1.3) or (1.4), whose speed is bounded by Theorem 1.1, cannot become pinned (at least) until every interval of length

$$
\left\lfloor\frac{\pi}{\cos ^{-1}\left(1-\frac{1}{2 \nu^{2}}\right)}\right\rfloor-1
$$

contains no more than two members of $\{0, n\} \cup n Z(u)$.

The paper is organized as follows. In Section 2 an elementary abstract result is presented that shows how to use variational techniques to estimate evolution speeds for a certain class of equations. In Section 3 some basic properties of (1.3) and (1.4) are discussed, in particular the existence of a conserved quantity for (1.4), called mass, and a dissipated quantity for (1.3) and (1.4), called energy. These properties will ensure that (for the proper choice of space and inner product) these equations fit into the framework of the preceding section. Section 4 contains the construction of initial data very close to $v$ with transition layers with very little excess energy. In Section 5, it is shown that there is a minimum energy cost per interface for any solution with a transition-layer structure, and Theorem 1.1 is then proved. Finally, in Section 6, Theorem 1.2 is proved.

2. Abstract Variational Method. The method that Bronsard and Kohn developed for detecting slowly-evolving solutions is based on a very simple idea: If motion is driven by energy dissipation and if there are solutions that move large distances without losing much energy, then they must move very slowly. In this section this principle is formulated as an abstract result general enough to apply to a large class of both ordinary and partial differential equations. In subsequent sections, we perform the delicate energy estimates necessary in order to apply this abstract result to the particular equations of interest.

Let $(X,\langle\cdot, \cdot\rangle)$ be an inner product space with induced norm $\|\cdot\|$, and let $M$ be a linear manifold in $X$, i.e., a translate of a linear subspace $\widetilde{M}$. Given a functional $F: M \rightarrow \mathbf{R}$ and a point $x \in M$, we will say that an element $y \in \widetilde{M}$ is the gradient of $F$ at $x$ on $M$ (and write $y=\nabla_{M} F(x)$ ) if for every differentiable curve $\gamma(t)$ in $M$ satisfying $\gamma\left(t_{0}\right)=x$,

$$
\left.\frac{d}{d t} F(\gamma(t))\right|_{t=t_{0}}=\left\langle y, \gamma^{\prime}\left(t_{0}\right)\right\rangle
$$

A gradient flow for $F$ on $M$ is a differential equation of the form

$$
\frac{d x}{d t}=-\kappa \nabla_{M} F(x)
$$


for some positive constant $\kappa$.

The following lemma, although quite simple, is really the core of the variational approach to dynamical metastability.

LEMMA 2.1. Let $x(t)$ be a solution of the gradient flow

$$
\frac{d x}{d t}=-\kappa \nabla_{M} F(x)
$$

on $M$ for $t \geq 0$. Then

$$
\|x(t)-x(0)\| \leq \sqrt{\kappa t(F(x(0))-F(x(t)))} .
$$

Proof. Note that

$$
\begin{aligned}
F(x(0))-F(x(t)) & =-\int_{0}^{t} \frac{d}{d \tau} F(x(\tau)) d \tau=-\int_{0}^{t}\left\langle\nabla_{M} F(x(\tau)), x^{\prime}(\tau)\right\rangle d \tau \\
& =\kappa \int_{0}^{t}\left\|\nabla_{M} F(x(\tau))\right\|^{2} d \tau \geq \frac{\kappa}{t}\left(\int_{0}^{t}\left\|\nabla_{M} F(x(\tau))\right\| d \tau\right)^{2}
\end{aligned}
$$

by Jensen's inequality. Thus,

$$
\begin{aligned}
\|x(t)-x(0)\| & =\left\|\int_{0}^{t} x^{\prime}(\tau) d \tau\right\| \leq \int_{0}^{t}\left\|x^{\prime}(\tau)\right\| d \tau \\
& =\kappa \int_{0}^{t}\left\|\nabla_{M} F(x(\tau))\right\| d \tau \leq \sqrt{\kappa t(F(x(0))-F(x(t)))} .
\end{aligned}
$$

(

3. Properties of Discrete Equations. Let $U_{n}$ be as in the Introduction, and define an inner product $\langle\cdot, \cdot\rangle_{2}$ on $U_{n}$ by

$$
\langle u, v\rangle_{2}=\frac{1}{n+1} \sum_{i=0}^{n} u_{i} v_{i},
$$

and set $m=(1,1, \ldots, 1)^{T}$. Two important real-valued functionals on $U_{n}$ are the mass $\langle m, \cdot\rangle_{2}$ and the energy $E$, defined by

$$
E(u)=\frac{1}{\nu} \sum_{i=0}^{n} W\left(u_{i}\right)+\frac{\nu}{2} \sum_{i=0}^{n-1}\left(u_{i+1}-u_{i}\right)^{2} .
$$

It will sometimes be necessary to refer to the energy on some set of consecutive integers other than $\{0,1, \ldots, n\}$. In those situations we will write

$$
E(u ; j, \ell)=\frac{1}{\nu} \sum_{i=j}^{\ell} W\left(u_{i}\right)+\frac{\nu}{2} \sum_{i=j}^{\ell-1}\left(u_{i+1}-u_{i}\right)^{2} .
$$

Let $\Delta$ be the linear operator on $U_{n}$ given by left-multiplication by the tridiagonal matrix

$$
\left[\begin{array}{ccccc}
-1 & 1 & & & \\
1 & -2 & 1 & & \\
& \ddots & \ddots & \ddots & \\
& & 1 & -2 & 1 \\
& & & 1 & -1
\end{array}\right]
$$


(We shall also refer to this matrix itself as $\Delta$.)

If $f: U_{n} \rightarrow U_{n}$ is now defined to be the map obtained by applying the real function $f$ componentwise, then (1.3) can be rewritten as a differential equation in $U_{n}$ :

$$
\dot{u}=\nu^{2} \Delta u-f(u) .
$$

Similarly, (1.4) can be simplified to the following pair of equations in $U_{n}$ :

$$
\begin{gathered}
\dot{u}=-n^{2} \Delta \mu(u), \\
\mu(u)=\nu^{2} \Delta u-f(u) .
\end{gathered}
$$

Note that $\Delta$ is self-adjoint with respect to $\langle\cdot, \cdot\rangle_{2}$ and that the null space of $\Delta$ is spanned by $m$. Let $\Delta^{\dagger}$ be the Moore-Penrose pseudo-inverse of $\Delta ;$ i.e., $\Delta^{\dagger} u$ satisfies $\Delta \Delta^{\dagger} u=u-\langle m, u\rangle_{2} m$ and $\left\langle m, \Delta^{\dagger} u\right\rangle_{2}=0$. Define $\langle\cdot, \cdot\rangle_{-1}$

$$
\langle u, v\rangle_{-1}=\langle H u, v\rangle_{2},
$$

where

$$
H=\frac{m m^{T}}{n+1}-\frac{\Delta^{\dagger}}{(n+1)^{2}} .
$$

We claim that this is an inner product on $U_{n}$. Its bilinearity is clear. Its symmetry follows from the fact that $\Delta^{\dagger}$ is self-adjoint with respect to $\langle\cdot, \cdot\rangle_{2}$, which, in turn, follows from the fact that $\Delta$ is self-adjoint with respect to $\langle\cdot, \cdot\rangle_{2}$. It remains only to show that it is positive definite, i.e., that $\langle u, u\rangle_{-1} \geq 0$ with equality only if $u=0$.

It is well-known that the eigenvalues of $-\Delta$ are

$$
\{2(1-\cos (j \pi /(n+1))): j=0,1, \ldots, n\} .
$$

Hence, the eigenvalues of $-\Delta^{\dagger}$ are

$$
\{0\} \cup\left\{[2(1-\cos (j \pi /(n+1)))]^{-1}: j=1, \ldots, n\right\},
$$

and the eigenvalues of $H$ are

$$
\{1\} \cup\left\{\left[2(n+1)^{2}(1-\cos (j \pi /(n+1)))\right]^{-1}: j=1, \ldots, n\right\} .
$$

This proves positive definiteness, so $\langle\cdot, \cdot\rangle_{-1}$ is indeed an inner product.

An application of Taylor's theorem to the eigenvalues of $H$ shows that they lie in the interval $\left[(2(n+1))^{-2}, 1\right]$. Thus, if we denote the induced norms corresponding to $\langle\cdot, \cdot\rangle_{2}$ and $\langle\cdot, \cdot\rangle_{-1},\|\cdot\|_{2}$ and $\|\cdot\|_{-1}$, respectively, then

$$
\frac{1}{2(n+1)}\|u\|_{2} \leq\|u\|_{-1} \leq\|u\|_{2} \text {. }
$$

Now, let $\gamma(t)$ be a curve in $U_{n}$. A calculation shows that

$$
\begin{aligned}
\frac{d}{d t} E(\gamma(t))= & \dot{\gamma}_{0}(t)\left[\nu^{-1} f\left(\gamma_{0}(t)\right)-\nu\left(-\gamma_{0}(t)+\gamma_{1}(t)\right)\right] \\
& +\sum_{i=1}^{n-1} \dot{\gamma}_{i}(t)\left[\nu^{-1} f\left(\gamma_{i}(t)\right)-\nu\left(\gamma_{i-1}(t)-2 \gamma_{i}(t)+\gamma_{i+1}(t)\right)\right] \\
& +\dot{\gamma}_{n}(t)\left[\nu^{-1} f\left(\gamma_{n}(t)\right)-\nu\left(\gamma_{n-1}(t)-\gamma_{n}(t)\right)\right]
\end{aligned}
$$


so if we let $\ell_{n}^{2}$ be the Hilbert space consisting of $U_{n}$ with inner product $\langle\cdot, \cdot\rangle_{2}$ then

$$
\nabla_{\ell_{n}^{2}} E(u)=(n+1)\left(\nu^{-1} f(u)-\nu \Delta u\right),
$$

and the discrete Allen-Cahn equation can be written

$$
\dot{u}=-\frac{\nu}{n+1} \nabla_{\ell_{n}^{2}} E(u) .
$$

Next, define $h_{n}^{-1}$ to be the Hilbert space consisting of $U_{n}$ with inner product $\langle\cdot, \cdot\rangle_{-1}$, and let $h_{n, m_{0}}^{-1}$ be the codimension one submanifold of $h_{n}^{-1}$ consisting of those elements with mass $m_{0}$. From (3.4) we have

$$
\frac{d}{d t} E(\gamma(t))=-\frac{n+1}{\nu}\langle\mu(\gamma(t)), \dot{\gamma}(t)\rangle_{2}
$$

where $\mu$ is as in (3.2). If $\gamma(t)$ is in $h_{n, m_{0}}^{-1}$ for all $t$ then $\langle m, \dot{\gamma}(t)\rangle_{2}=0$, so

$$
\begin{aligned}
\frac{d}{d t} E(\gamma(t)) & =-\frac{n+1}{\nu}\left\langle\left(-(n+1) m m^{T}+\Delta^{\dagger}\right) \Delta \mu(\gamma(t)), \dot{\gamma}(t)\right\rangle_{2} \\
& =\frac{(n+1)^{3}}{\nu}\langle H \Delta \mu(\gamma(t)), \dot{\gamma}(t)\rangle_{2}=\frac{(n+1)^{3}}{\nu}\langle\Delta \mu(\gamma(t)), \dot{\gamma}(t)\rangle_{-1} .
\end{aligned}
$$

Thus,

$$
\nabla_{h_{n, m_{0}}^{-1}} E(u)=\frac{(n+1)^{3}}{\nu} \Delta \mu(u)
$$

and the discrete Cahn-Hilliard equation can be written

$$
\dot{u}=-\frac{n^{2} \nu}{(n+1)^{3}} \nabla_{h_{n, m_{0}}^{-1}} E(u) .
$$

4. Efficient Initial Data. Since both (3.1) and (3.2) are gradient flows for E, Lemma 2.1 will eventually provide an estimate on the rate at which solutions evolve, if the energy decay can be estimated. In this section, initial data for the discrete equations are generated that are close to the discretized step function $v$ and that have efficient transition layers. Before stating the result, we introduce the quantity

$$
E_{\nu} \stackrel{\text { def }}{=} \inf \left\{E(z ;-\infty,+\infty): \lim _{i \rightarrow \pm \infty} z_{i} \operatorname{sgn} i=1\right\} .
$$

Lemma 4.1. There exists $u(0) \in U_{n}$ and a constant $B$ such that

$$
E(u(0)) \leq k E_{\nu}
$$

and

$$
\|u(0)-v\|_{\sigma}^{2} \leq B k \varepsilon, \quad \sigma=-1,2 .
$$

Furthermore,

$$
E_{\nu} \leq \min (2 \nu, 2 \sqrt{2} / 3)
$$


Proof. We construct the transition layers of $u(0)$ out of pieces of a profile realizing the infimum in the definition of $E_{\nu}$. First, we show that such a minimizer exists. Let $z^{(1)}, z^{(2)}, z^{(3)}, \ldots$ be a minimizing sequence; i.e., $E\left(z^{(j)} ;-\infty,+\infty\right) \rightarrow E_{\nu}$ as $j \rightarrow \infty$. Note that $E(\zeta ;-\infty,+\infty) \leq E\left(z^{(j)} ;-\infty,+\infty\right)$, where $\zeta=\min \left(1, \max \left(-1, z^{(j)}\right)\right)$. Also, note that all strictly decreasing segments of $\zeta$ have finite length and if $\xi$ is obtained from $\zeta$ by reversing each of its maximal decreasing segments then $E(\xi ;-\infty,+\infty) \leq E(\zeta ;-\infty,+\infty)$. Thus, without loss of generality we can assume that $z_{i}^{(j)}$ is contained in $[-1,1]$ and is monotone increasing in $i$. Because $E$ is translationally invariant, we may also assume that $z_{-1}^{(j)} \leq 0$ and $z_{0}^{(j)} \geq 0$. For fixed $i, z_{i}^{(j)}$ is a bounded sequence and, therefore, has a convergent subsequence. By a Cantor diagonalization process we can obtain a subsequence $z^{\left(j_{\ell}\right)}$ of $z^{(j)}$ that converges at every $i$. Call the pointwise limit $z$.

Note that $z_{i}$ is contained in $[-1,1]$ and is a nondecreasing function of $i$, and that $z_{-1} \leq 0$ and $z_{0} \geq 0$. We claim that $z$ has the correct limiting behavior at $\pm \infty$ so that $z$ is an admissible function for the minimization problem. To see why this must be so, observe that the monotonicity of $z^{(j)}$ implies that

$$
\frac{|i|}{\nu} W\left(z_{i}^{(j)}\right) \leq \max _{j} E\left(z^{(j)} ;-\infty,+\infty\right)
$$

so $z_{i}^{(j)} \operatorname{sgn} i \rightarrow 1$ uniformly in $j$ as $i \rightarrow \pm \infty$. Hence, the pointwise limit $z$ must have the same convergence property. Finally, note that

$$
\begin{aligned}
E(z ;-\infty,+\infty) & =\lim _{N \rightarrow \infty} E(z ;-N,+N)=\lim _{N \rightarrow \infty} \lim _{\ell \rightarrow \infty} E\left(z^{\left(j_{\ell}\right)} ;-N,+N\right) \\
& \leq \lim _{N \rightarrow \infty} \lim _{\ell \rightarrow \infty} E\left(z^{\left(j_{\ell}\right)} ;-\infty,+\infty\right)=E_{\nu} .
\end{aligned}
$$

This minimizer is not necessarily unique (even up to translation). Pick one such minimizer and call it $z^{\nu}$.

Let $d_{i}=\min \left\{|i-j|: v_{j} \neq v_{i}\right\}$. Then the initial data $u(0)$ will be given by

$$
u_{i}(0)=\left\{\begin{array}{cc}
z_{d_{i}-1}^{\nu} & \text { if } v_{i}=+1 \\
z_{-d_{i}}^{\nu} & \text { if } v_{i}=-1
\end{array}\right.
$$

Clearly, (4.1) holds. The distance from $u(0)$ to $v$ can be estimated as follows:

$$
\|u(0)-v\|_{2}^{2} \leq \frac{k}{n+1} \sum_{i=-\infty}^{+\infty}\left(1-\left|z_{i}^{\nu}\right|\right)^{2} \leq \frac{4 k}{n+1} \sum_{i=-\infty}^{+\infty} W\left(z_{i}^{\nu}\right) .
$$

Now pick $j \in\{0,1\}$ such that

$$
\sum_{i=-\infty}^{+\infty} W\left(z_{2 i+j}^{\nu}\right) \leq \frac{1}{2} \sum_{i=-\infty}^{+\infty} W\left(z_{i}^{\nu}\right)
$$

and let $\zeta_{i}=z_{2 i+j}^{\nu}$. Then by the optimality of $z^{\nu}$,

$$
\begin{aligned}
E_{\nu} & \leq \sum_{i=-\infty}^{+\infty}\left[\frac{1}{\nu} W\left(\zeta_{i}\right)+\frac{\nu}{2}\left(\zeta_{i+1}-\zeta_{i}\right)^{2}\right] \\
& \leq \frac{1}{2 \nu} \sum_{i=-\infty}^{+\infty} W\left(z_{i}^{\nu}\right)+\nu \sum_{i=-\infty}^{+\infty}\left(z_{i+1}^{\nu}-z_{i}^{\nu}\right)^{2}=2 E_{\nu}-\frac{3}{2 \nu} \sum_{i=-\infty}^{+\infty} W\left(z_{i}^{\nu}\right) .
\end{aligned}
$$


Hence,

$$
\sum_{i=-\infty}^{+\infty} W\left(z_{i}^{\nu}\right) \leq \frac{2 \nu}{3} E_{\nu}
$$

and

$$
\|u(0)-v\|_{2}^{2} \leq \frac{8 k \nu}{3(n+1)} E_{\nu}
$$

Now, evaluation of $E(\cdot ;-\infty,+\infty)$ at the admissible function $z_{i}=\tanh (i /(\nu \sqrt{2}))$ shows that $\limsup _{\nu \rightarrow \infty} E_{\nu} \leq 2 \sqrt{2} / 3$. Furthermore, by evaluating the energy of the admissable function $z_{i}=z_{2 i+j}^{2 \nu}$, where $j \in\{0,1\}$ is such that

$$
\sum_{i=-\infty}^{+\infty} W\left(z_{2 i+j}^{2 \nu}\right) \leq \frac{1}{2} \sum_{i=-\infty}^{+\infty} W\left(z_{i}^{2 \nu}\right)
$$

it can be seen that $E_{\nu} \leq E_{2 \nu}$ for any $\nu$. Thus, $E_{\nu} \leq 2 \sqrt{2} / 3$ for all $\nu$. Calculation of $E(z ;-\infty,+\infty)$ for $z_{i}=\operatorname{sgn}(i+1 / 2)$ completes the verification of (4.3). Substituting (4.3) into (4.5) yields (4.2), for $\sigma=2$. From (3.3), we also get (4.2) for $\sigma=-1$. $\square$

5. Minimum Energy of Transition-Layer Solutions. This section begins with three technical lemmas. The first lemma relates norm estimates to pointwise estimates.

Lemma 5.1. Suppose that $z \in U_{n}$ and that $S_{q}$ is a set of $q$ consecutive sites in the lattice. Then:

1. If $\|z\|_{2} \leq \delta$ then $z_{i} \leq \delta \sqrt{\frac{n+1}{q}}$ for some $i \in S_{q}$.

2. If $\|z\|_{-1} \leq \delta$ then $z_{i} \leq \delta \sqrt{\frac{13(n+1)^{3}}{q^{3}}}$ for some $i \in S_{q}$.

Proof. Suppose $\|z\|_{2} \leq \delta$. If $z_{i}>Z>0$ for all $i \in S_{q}$, then

$$
\delta^{2} \geq\|z\|_{2}^{2}=\frac{1}{n+1} \sum_{i=0}^{n} z_{i}^{2}>\frac{q Z^{2}}{n+1}
$$

so $Z<\delta \sqrt{\frac{n+1}{q}}$. This proves part 1 .

Suppose now that $\|z\|_{-1} \leq \delta$ and that $z_{i}>Z>0$ for all $i \in S_{q}$. Observe that $\Delta^{\dagger}=-\left(D^{-1} P\right)^{T} D^{-1} P$, where

$$
P=I-\frac{m m^{T}}{n+1}
$$

and $D^{-1}$ is the matrix with ones on and below the main diagonal and zeros above it. Then

$$
\|z\|_{-1}^{2}=\langle H z, z\rangle_{2}=\frac{\left(m^{T} z\right)^{2}}{(n+1)^{2}}+\frac{1}{(n+1)^{2}}\left\langle D^{-1} P z, D^{-1} P z\right\rangle_{2} .
$$

Setting $\beta=\left(m^{T} z\right) /(n+1)$ gives

$$
\|z\|_{-1}^{2}=\beta^{2}+\frac{1}{(n+1)^{3}} \sum_{i=0}^{n}\left(\sum_{j=0}^{i}\left(z_{j}-\beta\right)\right)^{2} \geq \min _{\hat{\alpha}, \hat{\beta}} F(\hat{\alpha}, \hat{\beta}),
$$


where

$$
F(\hat{\alpha}, \hat{\beta}) \stackrel{\text { def }}{=} \hat{\beta}^{2}+\frac{1}{(n+1)^{3}} \sum_{i=0}^{q}(\hat{\alpha}+i(Z-\hat{\beta}))^{2} .
$$

It is easy to verify that

$$
\min _{\hat{\alpha}, \hat{\beta}} F(\hat{\alpha}, \hat{\beta})=\frac{q^{3}+3 q^{2}+2 q}{12(n+1)^{3}+q^{3}+3 q^{2}+2 q} Z^{2},
$$

so

$$
Z \leq \delta \sqrt{\frac{12(n+1)^{3}}{q^{3}+3 q^{2}+2 q}+1} \leq \delta \sqrt{\frac{13(n+1)^{3}}{q^{3}}} .
$$

This proves part 2 . $\square$

The second lemma estimates the decay rate of an energy minimizer at one end of a finite lattice subject to a boundary condition at the other end.

Lemma 5.2. Given $z_{0} \in[0,1)$, let $z_{i},(i=1,2, \ldots, p)$, minimize $E(z-1 ; 0, p)$. Then

$$
z_{p} \leq \frac{2 z_{0}}{\left[1+\nu^{-1} \sqrt{\left(1-z_{0}\right)\left(2-z_{0}\right)}\right]^{p}} .
$$

Proof. Calculus implies that $z$ must satisfy

$$
z_{i}=2 z_{i+1}-z_{i+2}+\frac{1}{\nu^{2}} f\left(z_{i+1}-1\right)
$$

for $i=0,1, \ldots, p-2$, and

$$
z_{p-1}=z_{p}+\frac{1}{\nu^{2}} f\left(z_{p}-1\right)
$$

Also, $z_{i}$ must be in $\left[0, z_{0}\right]$ for each $i$. Thus, $f$ can be bounded from below to give the estimates

$$
z_{i} \geq \alpha z_{i+1}-z_{i+2}
$$

for $i=0,1, \ldots, p-2$, and

$$
z_{p-1} \geq(\alpha-1) z_{p}
$$

where

$$
\alpha=2+\nu^{-2}\left(1-z_{0}\right)\left(2-z_{0}\right) .
$$

For $i=0,1, \ldots, p+1$, define

$$
a_{i}=\frac{R^{1+i}-R^{1-i}}{R^{2}-1}
$$

where

$$
R=\frac{\alpha}{2}+\sqrt{\left(\frac{\alpha}{2}\right)^{2}-1}
$$


It is easy to see that $a_{i}$ satisfies the recurrence relation $a_{i}=\alpha a_{i-1}-a_{i-2}$ with initial conditions $a_{0}=0, a_{1}=1$. Also, note that $R>1$, so $a_{i} \geq 0$.

By induction on $i$ it can be shown that

$$
z_{0} \geq a_{i+1} z_{i}-a_{i} z_{i+1}
$$

for $j=0,1, \ldots, p-1$. In particular, (5.2) holds for $i=p-1$, so

$$
\begin{aligned}
z_{0} & \geq a_{p} z_{p-1}-a_{p-1} z_{p} \geq\left[a_{p}(\alpha-1)-a_{p-1}\right] z_{p}=\left(a_{p+1}-a_{p}\right) z_{p} \\
& =\frac{\left(R^{1+p}+R^{-p}\right) z_{p}}{R+1} \geq \frac{R^{p} z_{p}}{2} \geq \frac{\left[1+\nu^{-1} \sqrt{\left(1-z_{0}\right)\left(2-z_{0}\right)}\right]^{p} z_{p}}{2} .
\end{aligned}
$$

This implies (5.1).

The third lemma gives an upper bound for the minimal energy on a semi-infinite lattice.

Lemma 5.3. Given $z_{0} \in[0,1)$, there exists a sequence $z_{1}, z_{2}, \ldots$ converging to 0 such that

$$
E(z-1 ; 0, \infty)-\nu^{-1} W\left(z_{0}-1\right) \leq \nu z_{0}^{2} /(1+\nu \sqrt{2}) .
$$

Proof. Set $z_{i}=z_{0} R^{-i}$ where $R=1+\nu^{-1} \sqrt{2}$. A computation shows that

$$
E(z-1 ; 0, \infty)-\nu^{-1} W\left(z_{0}-1\right) \leq z_{0}^{2}\left[\frac{(R-1) \nu}{2(R+1)}+\frac{1}{\nu(R-1)(R+1)}\right]=\frac{\nu z_{0}^{2}}{1+\nu \sqrt{2}}
$$

These three lemmas can be combined to give a lower bound on $E(u)$ if $u$ is close to $v$ in $\ell_{n}^{2}$ or in $h_{n}^{-1}$.

LEMMA 5.4. Suppose

$$
\|u-v\|_{\sigma} \leq \delta
$$

where $\sigma=-1$ or 2 , (and $v$ is the discretized step function). Then there are positive constants $C_{2}, C_{3}, C_{4}$, and $C_{5}$ (depending on $k, r$, and $\delta$, but not on $\varepsilon$ or $n$ ) such that

$$
E(u) \geq k E_{\nu}-C_{5}\left[1+\frac{C_{2}}{n \varepsilon}\right]^{-C_{3} n+C_{4}}
$$

for $\delta \leq \frac{n r-1}{(n+1) \sqrt{r}}$ when $\sigma=2$, and for $\delta^{4} \leq \frac{(n r-1)^{9}}{169 r^{3}(n+1)^{9}}$ when $\sigma=-1$.

Proof. Suppose $\|u-v\|_{2} \leq \delta$ and that $v_{j}=-1$ and $v_{j+1}=+1$ for some $j$. Set $q=\lceil\delta(n+1) \sqrt{r}\rceil$ and apply Lemma 5.1 with $z=u-v$ to get $u_{\ell} \leq-1+z^{*}$ for some $\ell \in S_{q} \equiv\{j-q+1, j-q+2, \ldots, j\}$, where $z^{*}=\delta \sqrt{(n+1) / q}$. Similarly, $u_{\ell} \geq 1-z^{*}$ for some $\ell \in\{j+1, j+2, \ldots, j+q\}$. Set $p=\lceil n r\rceil-\lceil\delta(n+1) \sqrt{r}\rceil-1$. By Lemma 5.2,

$$
E(u ; j-p-q+1, j+q+p) \geq E(\phi ; j-p-q+1, j+q+p)
$$

for some $\phi$ satisfying

$$
\phi_{j-p-q+1} \leq-1+\frac{2 z^{*}}{\left[1+\nu^{-1} \sqrt{\left(1-z^{*}\right)\left(2-z^{*}\right)}\right]^{p}}
$$


and

$$
\phi_{j+q+p} \geq 1-\frac{2 z^{*}}{\left[1+\nu^{-1} \sqrt{\left(1-z^{*}\right)\left(2-z^{*}\right)}\right]^{p}} .
$$

(The function $\phi$ is obtained by replacing the tails of $u$ by the optimally decaying tails discussed in Lemma 5.2.) Thus, by Lemma 5.3,

$$
E(\phi ; j-p-q+1, j+q+p) \geq E_{\nu}-\frac{8 \nu\left(z^{*}\right)^{2}}{(1+\nu \sqrt{2})\left[1+\nu^{-1} \sqrt{\left(1-z^{*}\right)\left(2-z^{*}\right)}\right]^{2 p}},
$$

since otherwise $\phi$ could be extended to all of $\mathbf{Z}$ so as to be an admissible function for the minimization problem defining $E_{\nu}$ yet have energy less than $E_{\nu}$, which is a contradiction. By (5.5),

$$
E(u ; j-p-q+1, j+q+p) \geq E_{\nu}-\frac{8 \nu\left(z^{*}\right)^{2}}{(1+\nu \sqrt{2})\left[1+\nu^{-1} \sqrt{\left(1-z^{*}\right)\left(2-z^{*}\right)}\right]^{2 p}} .
$$

Clearly (5.6) also holds when $v_{j}=+1$ and $v_{j+1}=-1$.

If $\delta$ is as in the theorem statement, then as $j$ ranges over all jumps in $v,(5.6)$ estimates the energy of $u$ over disjoint sublattices of $\{0,1, \ldots, n\}$. We can, therefore, sum (5.6) over all $k$ jumps and simplify to get (5.4) for $\sigma=2$.

The proof for $\sigma=-1$ is similar. In this case, we can set $q=\left\lceil\left(169 \delta^{4} r^{3}\right)^{1 / 9}(n+1)\right\rceil$, $z^{*}=\delta \sqrt{13((n+1) / q)^{3}}$, and $p=\lceil n r\rceil-\left\lceil\left(169 \delta^{4} r^{3}\right)^{1 / 9}(n+1)\right\rceil-1$ to obtain $(5.4)$.

There are two more lemmas before the proof of the main result. The first of these gives a lower bound on the energy of a profile that is near \pm 1 at some sites and takes on values of the opposite sign at other sites. The second uses the first to establish a relationship between the distance between $v$ and $u$ in $\ell_{n}^{2}$ or $h_{n}^{-1}$ and the Hausdorff distance between their zero sets, if it is assumed that $u$ has efficient transition layers.

Lemma 5.5. Suppose $\left|z_{0}\right| \geq 1 / \sqrt{3}$ and $z_{0} z_{j} \leq 0$ for some $j \in \mathbf{N}$. Then

$$
E(z ; 0, j)-\nu^{-1} W\left(z_{0}\right) \geq \min (2 \nu / 3,(\sqrt{6}) / 9) .
$$

Furthermore, if $\left|z_{j}\right| \geq 1 / \sqrt{3}$ then

$$
E(z ; 0, j)-\nu^{-1}\left(W\left(z_{0}\right)+W\left(z_{j}\right)\right) \geq \min (2 \nu / 3,(\sqrt{6}) / 9) .
$$

Proof. Without loss of generality, assume $z_{0}<0$. Set $\widehat{W}(u)$ to be $1 / 9$ for $|u|<1 / \sqrt{3}$ and 0 elsewhere. Note that, since $\widehat{W}(u) \leq W(u)$, to prove $(5.7)$ and (5.8) it suffices to show that

$$
\widehat{E} \geq \min (2 \nu / 3,(\sqrt{6}) / 9),
$$

where

$$
\widehat{E}=\widehat{E}\left(z_{0}, z_{1}, \ldots, z_{j} ; j\right)=\sum_{i=1}^{j}\left[\frac{1}{\nu} \widehat{W}\left(z_{i}\right)+\frac{\nu}{2}\left(z_{i}-z_{i-1}\right)^{2}\right]
$$


It is not hard to see that if $j$ and $\left(z_{0}, z_{1}, \ldots z_{j}\right)$ minimize $\widehat{E}$ then either $z_{i}=(2 i-1) / \sqrt{3}$ and $j=1$, or $z_{i}=(i / j-1) / \sqrt{3}$ and $j \geq 1$. In the first case, $\widehat{E}=2 \nu / 3$, and in the second case

$$
\widehat{E}=\min _{j \in \mathbf{N}}\left[\frac{j}{9 \nu}+\frac{\nu}{6 j}\right] \geq \min _{x \in \mathbf{R}^{+}}\left[\frac{x}{9}+\frac{1}{6 x}\right]=\frac{\sqrt{6}}{9} .
$$

Thus, (5.9) holds. $\square$

LEMmA 5.6. If $\|u-v\|_{2} \leq \delta$ and $E(u) \leq k E_{\nu}$ then

$$
d(Z(u), Z(v)) \leq \frac{\left\lfloor 6 \delta^{2} k(n+1)\right\rfloor+1 / 2}{n} .
$$

If $\|u-v\|_{-1} \leq \delta$ and $E(u) \leq k E_{\nu}$ then

$$
d(Z(u), Z(v)) \leq \frac{\left\lfloor\left(78 \delta^{2} k\right)^{1 / 3}(n+1)\right\rfloor+1 / 2}{n} .
$$

Proof. Suppose $\|u-v\|_{2} \leq \delta$ and $E(u) \leq k E_{\nu}$, and set $q=\left\lfloor 6 \delta^{2} k(n+1)\right\rfloor+1$, and $z^{*}=\delta \sqrt{(n+1) / q} \leq 1-1 / \sqrt{3}$. For each discontinuity in $\tilde{v}$, Lemma 5.1 shows that $u$ takes on a value less than $-1+z^{*}$ within the first $q$ lattice sites on one side and a value greater than $1-z^{*}$ within the first $q$ lattice sites on the opposite side. By Lemma 5.3, the energy of $u$ between these two sites is no less than $E_{\nu}-2 \nu\left(z^{*}\right)^{2} /(1+\nu \sqrt{2})$. Considering all $k$ discontinuities of $\tilde{v}$, we see that an energy of at least

$$
k E_{\nu}-2 k \nu\left(z^{*}\right)^{2} /(1+\nu \sqrt{2})
$$

is expended within $q$ sites of the discontinuities. If there were a zero of $u$ more than $q$ lattice sites away from a discontinuity in $\tilde{v}$ then by Lemma 5.5 the extra energy needed to achieve this would be at least $\min (2 \nu / 3,(\sqrt{6}) / 9)$. Adding this to $(5.12)$ would give a contradiction, in light of (4.3). Hence,

$$
\max _{x \in Z(u)} d(x, Z(v)) \leq \frac{q-1 / 2}{n} .
$$

Also, the Intermediate Value Theorem implies that

$$
\max _{x \in Z(v)} d(x, Z(u)) \leq \frac{q-1 / 2}{n} .
$$

Combining (5.14) and (5.13) yields (5.10).

If $\|u-v\|_{-1} \leq \delta$, then setting $q=\left\lfloor\left(78 \delta^{2} k\right)^{1 / 3}(n+1)\right\rfloor+1$ and reasoning as above, we obtain (5.11).

Proof of Theorem 1.1. We give the proof for (1.3); the result for (1.4) can be handled similarly. Pick $n_{0}=n_{0}(\rho)$ large enough that

$$
0<\frac{2 \rho n_{0}-1}{12\left(n_{0}+1\right)} \leq \frac{\left(n_{0} \rho-1\right)^{2}}{\rho\left(n_{0}+1\right)^{2}}
$$

and set $\delta^{2}=\left(2 \rho n_{0}-1\right) /\left(12\left(n_{0}+1\right) k\right)$. Pick $\varepsilon_{0}=\varepsilon_{0}(\rho, k)$ small enough that $4 B k \varepsilon_{0} \leq \delta^{2}$. Assume $n \geq n_{0}$ and $\varepsilon \leq \varepsilon_{0}$, and apply Lemma 4.1 to get initial data $u(0) \in U_{n}$ satisfying $E(u(0)) \leq k E_{\nu}$ and $\|u(0)-v\|_{2}^{2} \leq B k \varepsilon$, and let $u(t)$ be the corresponding solution of 
(3.1). Suppose $d(Z(v), Z(u(t))) \geq \rho$. Then since $E(u(t)) \leq E(u(0))$, the estimate (5.10) in Lemma 5.6 implies that $\|u(t)-v\|_{2} \geq \delta$. Also by the choice of $n_{0}$ and $\varepsilon_{0},\|u(0)-v\|_{2} \leq \delta / 2$, so there is a first $T \in(0, t]$ such that $\|u(T)-v\|_{2}=\delta$.

By our choice of $n_{0}$, we are able to apply Lemma 5.4 to obtain

$$
E(u(T)) \geq k E_{\nu}-C_{5}\left[1+\frac{C_{2}}{n \varepsilon}\right]^{-C_{3} n+C_{4}} .
$$

Thus,

$$
\|u(T)-u(0)\|_{2} \geq\|u(T)-v\|_{2}-\|u(0)-v\|_{2} \geq \delta / 2
$$

and

$$
E(u(0))-E(u(T)) \leq \frac{C_{5}}{\left[1+\frac{C_{2}}{n \varepsilon}\right]^{C_{3} n-C_{4}}} .
$$

Applying Lemma 2.1 to (3.5) and using (5.15) and (5.16) yields

$$
T \geq \frac{1}{C_{1} \varepsilon}\left(1+\frac{C_{2}}{n \varepsilon}\right)^{C_{3} n-C_{4}}
$$

口

\section{Properties of Equilibria.}

Proof of Theorem 1.2. Because it is an equilibrium, $u$ must satisfy

$$
\nu^{2} \Delta u=f(u)+\alpha m
$$

for some constant $\alpha$. Extend $u$ to $\ell=n+1, \ldots, 2 n+1$ by the formula $u_{\ell}=u_{2 n+1-\ell}$, and then extend it to be a $(2 n+2)$-periodic function on all of $\mathbf{Z}$. Define $w_{\ell}=u_{\ell}-u_{\ell-1}$, and note that (6.1) implies that

$$
\nu^{2}\left(w_{\ell-1}-2 w_{\ell}+w_{\ell+1}\right)=f\left(u_{\ell}\right)-f\left(u_{\ell-1}\right),
$$

for all $\ell \in \mathbf{Z}$. Since $f^{\prime} \geq-1$, the Mean Value Theorem applied to (6.2) yields

$$
w_{\ell+1} \geq\left(2-\frac{1}{\nu^{2}}\right) w_{\ell}-w_{\ell-1}
$$

if $w_{\ell} \geq 0$.

Now suppose $u_{i}$ is a local minimum, so $w_{i} \leq 0$ and $w_{i+1} \geq 0$. We shall prove that, in fact, $w_{i+j} \geq 0$ for $j=1,2, \ldots, p$, where

$$
p=\left\lfloor\frac{\pi}{\cos ^{-1}\left(1-\frac{1}{2 \nu^{2}}\right)}\right\rfloor .
$$

To do this we will use the auxiliary sequence $a_{\ell}=\frac{\sin \ell \theta}{\sin \theta}$, where $\theta=\cos ^{-1}\left(1-1 /\left(2 \nu^{2}\right)\right)$. Since $\nu \geq 1 / \sqrt{2}, \theta \in(0, \pi / 2]$, so this sequence is well-defined. Note that $a_{0}=0, a_{1}=1$, $a_{2}=2-1 / \nu^{2}$, and $a_{\ell+1}=a_{2} a_{\ell}-a_{\ell-1}$. Note also that $a_{\ell} \geq 0$ for $\ell=0,1, \ldots, p$.

Now, let $P_{j}$ be the proposition that $w_{i+1} \geq 0, w_{i+2} \geq 0, \ldots, w_{i+j} \geq 0$. We will prove $P_{j}$ for $j=1, \ldots, p$ by induction on $j$. Also, let $Q_{j, \ell}$ be the proposition that $w_{i+j+1} \geq$ 
$a_{\ell} w_{i+j+2-\ell}-a_{\ell-1} w_{i+j+1-\ell}$. For fixed $j<p$, we will prove $Q_{j, \ell}$ for $\ell=1, \ldots, j+1$ by induction on $\ell$.

$P_{1}$ is the statement $w_{i+1} \geq 0$, which is true by assumption. Suppose $P_{j}$ is true, for some $j<p$. Fix $j$, and note that $Q_{j, 1}$ is the statement $w_{i+j+1} \geq w_{i+j+1}$, which is, of course, true. Suppose $Q_{j, \ell}$ holds for some $\ell \leq j$. Since $i+1 \leq i+j+1-\ell \leq i+j, P_{j}$ implies that $w_{i+j+1-\ell} \geq 0$. Also, $a_{\ell} \geq 0$, since $\ell \leq j<p$. Thus, (6.3) and $Q_{j, \ell}$ imply that

$$
\begin{aligned}
w_{i+j+1} & \geq a_{\ell} w_{i+j+2-\ell}-a_{\ell-1} w_{i+j+1-\ell} \geq a_{\ell}\left[\left(2-\nu^{-2}\right) w_{i+j+1-\ell}-w_{i+j-\ell}\right]-a_{\ell-1} w_{i+j+1-\ell} \\
& =\left(a_{\ell} a_{2}-a_{\ell-1}\right) w_{i+j+1-\ell}-a_{\ell} w_{i+j-\ell}=a_{\ell+1} w_{i+j+2-(\ell+1)}-a_{(\ell+1)-1} w_{i+j+1-(\ell+1)},
\end{aligned}
$$

so $Q_{j, \ell+1}$ holds. By induction, $Q_{j, \ell}$ holds for $\ell=1, \ldots, j+1$. In particular, we have $Q_{j, j+1}$ :

$$
w_{i+j+1} \geq a_{j+1} w_{i+1}-a_{j} w_{i} \geq 0,
$$

so $P_{j+1}$ holds. By induction, $P_{j}$ holds for $j=1, \ldots, p$. In particular, we have $P_{p}$ :

$$
w_{i+1} \geq 0, \ldots, w_{i+p} \geq 0,
$$

verifying the earlier claim. This implies that if $u_{j}$ is a local maximum for (the extended) $u$ and $j>i$ then $j \geq i+p-1$, so $|i-j| \geq p-1$. A similar result holds if $j<i$. Clearly, the same estimate holds for the original $u \in U_{n}$, so the proof of (1.5) is complete. The second half of Theorem 1.2 is an immediate consequence.

7. Acknowledgments. Work on this problem was initiated while the first author was with the Center for Dynamical Systems and Nonlinear Studies at Georgia Institute of Technology and the second author was with the Department of Mathematics and Statistics at Simon Fraser University. We wish to thank the referees for several helpful suggestions.

\section{REFERENCES}

[1] N. D. Alikakos, P. W. Bates, And G. Fusco, Slow motion for the Cahn-Hilliard equation in one space dimension, J. Differential Equations, 90 (1991), pp. 81-135.

[2] N. D. Alikakos And G. Fusco, Slow dynamics for the Cahn-Hilliard equation in higher space dimensions part I: Spectral estimates. preprint.

[3] - Slow dynamics for the Cahn-Hilliard equation in higher space dimensions part II. in preparation.

[4] S. Allen And J. CAhn, A microscopic theory for antiphase boundary motion and its application to antiphase domain coarsening, Acta. Metall., 27 (1979), pp. 1084-1095.

[5] P. W. Bates And J. P. Xun, Metastable patterns for the Cahn-Hilliard equation, J. Differential Equations, 111 (1994), pp. 421-457.

[6] J. BELl, Some threshold results for models of myelinated nerves, Math. Biosciences, 54 (1981), pp. 181-190.

[7] J. Bell And C. Cosner, Threshold behavior and propagation for nonlinear differential-difference systems motivated by modeling myelinated axons, Quart. Appl. Math., 42 (1984), pp. 1-114.

[8] L. Bronsard And R. V. Kohn, On the slowness of phase boundary motion in one space dimension, Comm. P. A. Math., 43 (1990), pp. 983-998.

[9] - Motion by mean curvature as the singular limit of Ginzburg-Landau dynamics, J. Differential Equations, 90 (1991), pp. 211-237.

[10] J. W. CAHn, Theory of crystal growth and interface motion in crystalline materials, Acta Metall., 8 (1960), pp. 554-562.

[11] — On spinodal decomposition, Acta Metall., 9 (1961), pp. 795-801.

[12] J. W. Cahn, S. N. Chow, And E. S. VAN Vleck, Spatially discrete nonlinear diffusion equations. Rocky Mountain J. Math., to appear.

[13] J. W. CAhn AND J. E. Hilliard, Free energy of a nonuniform system I. interfacial free energy, Journal of Chemistry and Physics, 28 (1958), pp. 258-267. 
[14] J. Carr And R. L. Pego, Metastable patterns in solutions of $u_{t}=\varepsilon^{2} u_{x x}-f(u)$, Comm. P. A. Math., 42 (1989), pp. 523-576.

[15] X. Chen, Generation and propagation of interfaces for reaction-diffusion equations, J. Differential Equations, 96 (1992), pp. 116-141.

[16] H. Chi, J. Bell, AND B. HASSARD, Numerical solution of a nonlinear advance-delay-differential equation from nerve conduction theory, J. Math. Biol., 24 (1986), pp. 583-601.

[17] H. E. Cook, D. de Fontaine, And J. E. Hilliard, A model for diffusion on cubic lattices and its application to the early stages of ordering, Acta Met., 17 (1969), pp. 765-773.

[18] P. Demottoni and M. Schatzman, Evolution géométrique d'interfaces, C. R. Acad. Sci. Paris, Série I, 309 (1989), pp. 453-458.

[19] - Development of interfaces in $R^{N}$, Proc. Roy. Soc. Edinburgh, Sec. A, 116 (1990), pp. $207-220$.

[20] Q. Du AND R. A. NiCOlaides, Numerical analysis of a continuum model of phase transition, SIAM J. Numer. Anal., 28 (1991), pp. 1310-1322.

[21] C. M. Elliott And D. A. French, Numerical studies of the Cahn-Hilliard equation for phase separation, IMA J. Appl. Math., 38 (1987), pp. 97-128.

[22] C. M. Elliott And A. M. Stuart, The global dynamics of discrete semilinear parabolic equations, SIAM J. Numer. Anal., 30 (1993), pp. 1622-1663.

[23] D. EsteP, An analysis of numerical approximations of metastable soutions of the bistable equation, Nonlinearity, 7 (1994), pp. 1445-1462.

[24] L. C. Evans, H. M. Soner, And P. E. Souganidis, Phase transitions and generalized motion by mean curvature, Comm. Pure Appl. Math., 45 (1992), pp. 1097-1123.

[25] G. Fusco, A geometric apporach to the dynamics of $u_{t}=\varepsilon^{2} u_{x x}-f(u)$, in Proceedings of the Stuttgart conference in honor of J. K. Hale, K. Kirchgässner, ed., Springer-Verlag, 1990, pp. 53-73.

[26] G. Fusco And J. K. Hale, Slow-motion manifolds, dormant instability, and singular perturbations, J. Dynamics and Diff. Eqns., 1 (1989), pp. 75-94.

[27] C. P. Grant, Spinodal decomposition for the Cahn-Hilliard equation, Comm. in P.D.E., 18 (1993), pp. 453-490.

[28] — Slow motion in one-dimensional Cahn-Morral systems, SIAM J. Math. Anal., 26 (1995), pp. 21-34.

[29] J. K. HALE, Numerical dynamics, in Chaotic Numerics, P. E. Kloeden and K. J. Palmer, eds., vol. 172 of Contemporary Mathematics, American Mathematical Society, Providence, Rhode Island, 1994, pp. $1-30$.

[30] M. Hillert, A solid-solution model for inhomogeneous systems, Acta Met., 9 (1961), pp. 525-535.

[31] T. Ilmanen, Convergence of the Allen-Cahn equation to Brakke's motion by mean curvature, J. Differential Geom., 38 (1993), pp. 417-461.

[32] J. P. KeEner, Propagation and its failure in coupled systems of discrete excitable cells, SIAM J. Appl. Math., 47 (1987), pp. 556-572.

[33] W. R. McKinney, Optimal Error Estimates for High Order Runge-Kutta Methods Applied to Evolutionary Equations, PhD thesis, Univeristy of Tennessee, 1989.

[34] P. Sternberg, The effect of a singular perturbation on nonconvex variational problems, Arch. Rational Mech. Anal., 101 (1988), pp. 209-260.

[35] B. ZINnER, Stability of traveling wavefronts for the discrete Nagumo equation, SIAM J. Math. Anal., 22 (1991), pp. 1016-1020.

[36] — Existence of traveling wavefront solutions for the discrete Nagumo equation, J. Differential Equations, 96 (1992), pp. 1-27.

[37] - Wavefronts for a cooperative tridiagonal system of differential equations, J. Dynamics and Diff. Eqns., 5 (1993), pp. 359-373. 